\title{
Lateral oblique approach for internal jugular vein catheterization: Randomized comparison of oblique and short-axis view of ultrasound-guided technique
}

\author{
(D) Onur Balaban, ${ }^{* 1}$ (1) Tayfun Aydin, ${ }^{1}$ (1) Ahmet Musmul² \\ ${ }^{1}$ Department of Anesthesiology and Pain, Dumlupinar University Faculty of Medicine, Kutahya, Turkey \\ ${ }^{2}$ Department of Medical Services and Techniques, Eskisehir Osmangazi University Vocational School of Health Services, Eskisehir, Turkey
}

\begin{abstract}
OBJECTIVE: The lateral oblique approach is a novel needle-in-plane technique for ultrasound-guided catheterization of the internal jugular vein. In this study, we aimed to compare the oblique approach with the classical short-axis technique for facilitating the procedure and reduction of mechanical complications.

METHODS: This research was planned as a prospective study. Eighty-four open-heart surgery patients requiring a central venous catheter were randomly allocated into two groups: Oblique approach group $(n=42)$ and short-axis group $(n=42)$. Time to cannulate, the number of necessary puncture attempts, and frequency of carotid artery puncture, hematoma, puncture site bleeding, pneumothorax, and hemothorax in each group were recorded. Visualization of the vein and the needle using ultrasound were also evaluated by a subjective scale.

RESULTS: The patient's characteristics were comparable between the two groups. The mean time of catheterization was $52.00 \pm 70.18$ seconds in the oblique approach group and $40.76 \pm 49.30$ seconds in short-axis group. The mean number of needle puncture attempts was $1.21 \pm 0.61$ in oblique approach and $1.12 \pm 0.50$ in short-axis group. The results did not differ significantly. There was an improved visualization of the needle in the oblique approach group, but this was not proved as statistically significant.
\end{abstract}

CONCLUSION: The results of our study suggest that the lateral oblique approach is a safe and effective technique, which can be a strong alternative to the classical short-axis technique for ultrasound-guided catheterization of the internal jugular vein.

Keywords: Central venous catheterization; internal jugular vein; lateral oblique approach; ultrasound.

Cite this article as: Balaban O, Aydın T, Musmul A. Lateral oblique approach for internal jugular vein catheterization: Randomized comparison of oblique and short-axis view of ultrasound-guided technique. North Clin Istanb 2020;7(1):11-17.

$U_{t}^{\prime}$ ltrasonography is recommended and has been found superior to the classic landmark technique for central venous catheterization $[1,2]$. Agency for Healthcare Research and Quality report listed ultrasound (US) guidance for placement of central venous catheters (CVC) as one of the top patient safety practices and the use of the US during catheterization was recommended by the National Institute of Clinical Ex- cellence since 2002 [3, 4]. Ultrasound-guided central venous catheter placement decreases complications and decreases catheter placement attempts [5]. Real-time two- dimensional ultrasound guidance was found in association with decreased risks of cannulation failure, arterial puncture, hematoma, and hemothorax, compared to the landmark technique [6].

There are many approaches and techniques for ultra-

*Here's the updated affiliation: Department of Anesthesiology and Pain, Kutahya Health Sciences University, Kutahya, Turkey.

Received: December 11, 2017 Accepted: February 19, 2019 Online: July 03, 2019

Correspondence: Dr. Onur BALABAN. Kutahya Saglik Bilimleri Universitesi, Evliya Celebi Egitim Arastirma Hastanesi, Okmeydani Caddesi, 43040 Merkez, Kutahya, Turkey.

Tel: +9027423166 60 e-mail: obalabandr@gmail.com

(c) Copyright 2020 by Istanbul Provincial Directorate of Health - Available online at www.northclinist.com 
sound-guided central venous catheterization $[1,6]$. Catheterization of the internal jugular vein (IJV) is usually preferred because of its anatomical position and large diameter in the Trendelenburg position [7]. The puncture may be performed using an in-plane approach with a long axis (LA) visualization or out of plane approach with a short-axis visualization of IJV. Although the short-axis (transverse) visualization is mostly used by practitioners for internal jugular vein catheterization, this technique was found suboptimal considering the lack of prevention of posterior wall puncture [8]. The in-plane (long axis) technique for the needle guidance ensures the view of the entire needle, including tracking of the needle tip [9].

The lateral oblique visualization of the IJV is a novel approach and different from previous techniques, which was primarily offered by Phelan, and Hagerty for ultrasound-guided IJV catheterization [10]. This approach may be an alternative to short-axis and long-axis approaches for the US-guided IJV catheterization. Oblique visualization provides a longer view of the IJV along with the carotid artery. In this approach, real-time visualization of the entire needle, including the tip is possible. Compared to the long axis view of the IJV, the lateral oblique approach ensures a wider lateral space at the neck for needle insertion that may facilitate needle insertion.

The present study aims to evaluate the needle-in-plane technique with oblique visualization of the internal jugular vein in comparison to the classical short-axis (needle out of plane) technique for catheterization of the internal jugular vein. Our hypothesis was time for catheterization, the number of cannulation attempts and complications during catheterization may be reduced when oblique approach is used. We compared these two techniques in regard of catheterization times, the number of puncture attempts and the frequency of complications.

\section{MATERIALS AND METHODS}

The present research was planned as a prospective randomized study. IRB approval (Kocaeli University, KOU KAEK 2015/1) was obtained. Written informed consent was obtained from all participants before they were included in this study. Patients who were scheduled for an open-heart surgery and required a central venous catheter were included in this study. Exclusion criteria were as follows: patients requiring an emergency openheart surgery, patients having a history of previous surgical intervention at the catheterization site, or with distorted neck anatomy and patients having a body mass index $>40.00 \mathrm{~kg} / \mathrm{m}^{2}$. Age $<18$ years, cervical trauma with present neck immobilization and patients with signs of infection or subcutaneous hematoma at the puncture site were also excluded. A computer-generated random number list was used and the patients were randomly assigned into two groups: Oblique approach group (OA) and short-axis group (SA).

After monitoring invasive arterial pressure, electrocardiogram and pulse oximetry, induction of general anesthesia was achieved using fentanyl $5 \mu \mathrm{g} / \mathrm{kg}$ intravenous, thiopental $5 \mathrm{mg} / \mathrm{kg}$ intravenous and rocuronium $0.6 \mathrm{mg} / \mathrm{kg}$ intravenous. Anesthesia was maintained with Sevoflurane $2 \%$ and oxygen $50 \%$. The catheterizations were performed under ultrasound guidance using a linear array $12 \mathrm{MHz}$ ultrasound probe (Logic P5, GE Healthcare, Milwaukee WI, USA). Before catheterization, internal jugular vein at both sides of the patients was scanned throughout the neck and abnormalities were recorded. The other side was preferred for cannulation if any abnormality was detected. Patients were placed in the supine position with the head rotated slightly (about $30^{\circ}$ ) to the opposite side of the catheterization site in a neutral position (not Trendelenburg). The skin was prepared with povidone-iodine and the US probe was covered with a sterile cap and sterile gel was used as a coupling agent. All catheterizations were performed by the same attending physician (O.B.) using a single-performer technique with the same operator handling the US probe and the needle. Catheterizations were performed using the Seldinger technique, with an $18-\mathrm{G} 6.5 \mathrm{~cm}$ introducer needle (Arrow International, Reading, PA, USA).

The oblique view is described as placing the US probe at 45 degrees angle to ensure an in-plane visualization of the needle while approaching the target vessel, which is inserted from lateral to medial [10]. The patients in the oblique approach group were cannulated using needle inplane technique. In this group, the ultrasound probe was placed on the jugular vein at mid-neck level to capture a transverse cross-sectional image of the carotid artery and IJV together. Once the short-axis view is obtained, the probe is rotated 45 degrees clockwise (or counter clockwise if left IJV is chosen), with the orientation marker medially (or lateral if left IJV is attempted). An oblique view of the IJV and carotid artery was visualized, which is sonographically seen as oval structures posterior to the sternocleidomastoid muscle. The needle was inserted from the lateral aspect of the probe within the plane of the US beam underneath the footprint and advanced medially (Fig. 1). The needle was visualized in real-time 


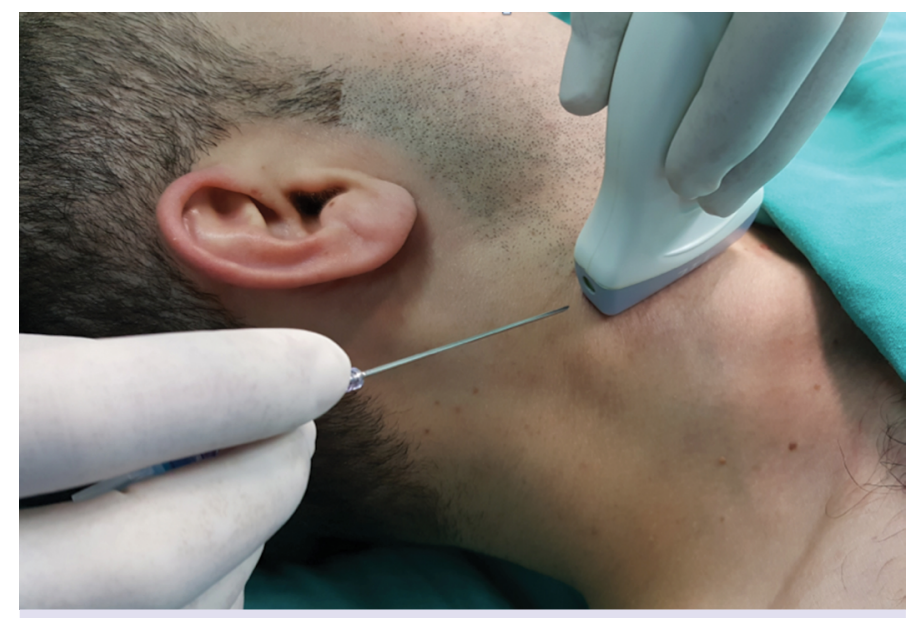

FIGURE 1. The position of the ultrasound probe and the needle during catheterization using the oblique approach.

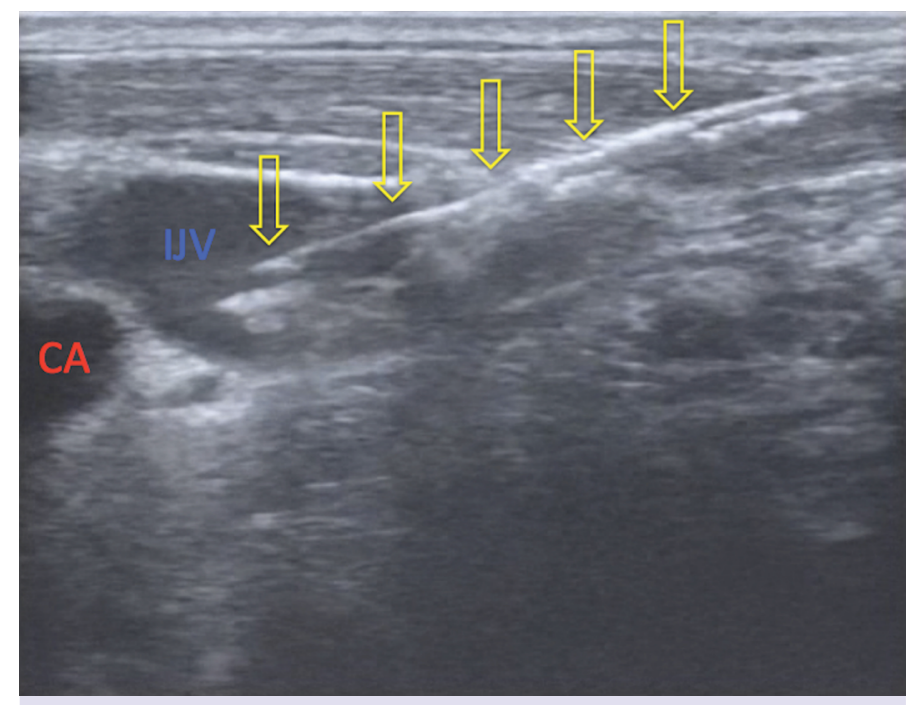

FIGURE2. Ultrasonographic visualization of the internal jugular vein and the needle using the oblique approach. The internal jugular vein (IJV) and the carotid artery (CA) are visualized obliquely. The whole needle (shown by arrows) can be visualized in real-time during the procedure.

while it was being advanced forward and entering the IJV (Fig. 2). Then, the ultrasound probe was removed and a three-port central catheter (Arrow International, Reading, PA, USA) was placed according to the standard Seldinger technique. A syringe-free technique was used for the insertion of the needle. We did not attach a syringe to the needle and we did not aspirate blood during insertion of the needle. Instead of that, we inserted an open-ended needle and observe the tip of the needle under ultrasound guidance. When the tip of the needle was visualized in the IJV, the guide wire was inserted through

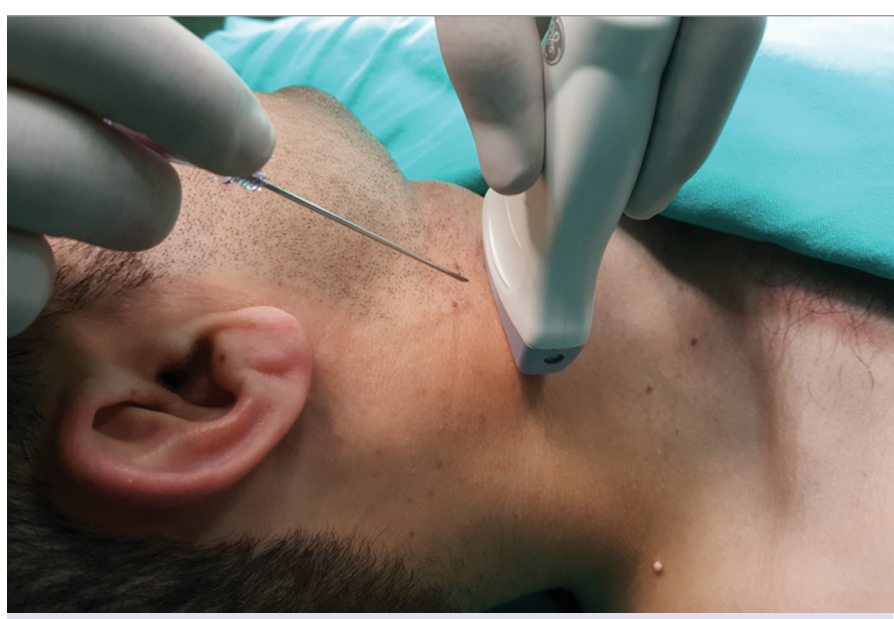

FIGURE 3 . The position of the US probe and the needle during catheterization using short-axis technique.

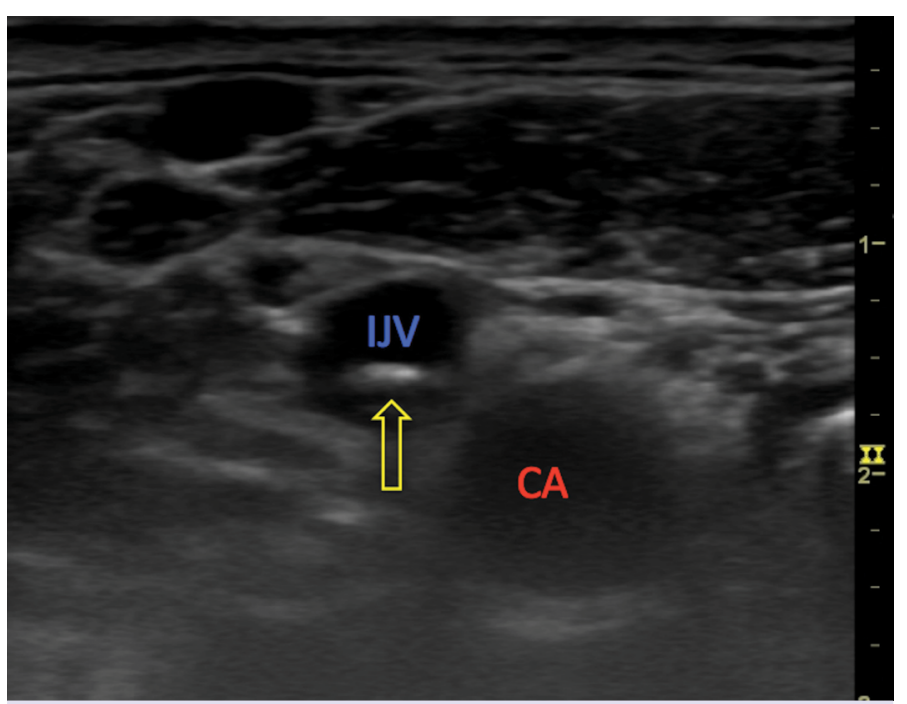

FIGURE 4. Ultrasonographic visualization of the internal jugular vein and the needle using a short-axis approach. A crosssectional image of the internal jugular vein (IJV) and the carotid artery (CA) is visualized. The needle could be visualized as an echogenic dot in the vein (arrow).

the needle into the vein. We did not wait to observe blood at the end of the needle. We immediately inserted the guide wire to prevent air embolism.

In the short-axis group, the US probe was placed transversally over the neck to capture a transverse, shortaxis view of the IJV and carotid artery. The needle was inserted from the midpoint of the probe towards the center of IJV using out of plane technique (Fig. 3) with a syringe attached at the end. The tip of the needle is visualized as a small hyper-echoic dot when it crossed the wall of the vein (Fig. 4). When blood was aspirated, the 
guide wire was inserted through the needle into the vein. The insertion of a guide wire is verified by the US in the IJV after the procedures.

Demographic characteristics (age, gender, BMI) and type of operation were recorded. The primary outcomes were the catheterization time, the number of puncture attempts for successful placement and potential complications during catheterization. Expected mechanical complications were as follows: carotid artery puncture, local hematoma formation (which was identified by ultrasound), puncture site bleeding, pneumothorax, and hemothorax. Carotid artery puncture was defined as the observation of pulsatile blood reflux through the needle. Mechanical complications were diagnosed by ultrasonography and clinical evaluation. The side of catheterization was also recorded.

Catheterization time was defined as the time starting from the first skin puncture until the placement of the guide wire into the vein without resistance and withdrawal of the needle. As all of the catheters were inserted using the Seldinger technique, only cannulation of the vein with a guide wire was determined as time of the procedure, we did not add the remainder of the procedure in the procedure time. The difficulty of US visualization of both the vein and the needle was recorded and classified as: 1.easy to visualize 2.moderate 3.difficult to visualize 4.impossible.

Data were recorded in an electronic spreadsheet (Microsoft Excel, Microsoft Corporation, Redmond, WA). All data were analyzed using SPSS 21 (SPSS Inc., Chicago, IL, USA) package program after transferring to SPSS format. Continuous quantitative data were presented as number, mean \pm standard deviation. Qualitative variables were expressed as median, $25^{\text {th }}$ and $75^{\text {th }}$ percentiles. Continuous variables consisted of independent measure and normal distribution was analyzed with independent samples t-test. Mann-Whitney rank-sum test was applied to the data without normal distribution. Chi-square tests were applied to the categorical data. $\mathrm{p}<0.05$ was considered significant.

\section{RESULTS}

A total of 84 patients was enrolled in this study. The patient's characteristics are summarized in Table 1 . There were no significant differences between the two groups concerning age, weight, height, BMI, gender and side of catheterization.

\section{TABLE 1. Patient characteristics}

\begin{tabular}{|c|c|c|c|}
\hline & \multicolumn{2}{|c|}{ Group } & \multirow[t]{2}{*}{$p$} \\
\hline & Oblique & Short axis & \\
\hline \multicolumn{4}{|l|}{ Age (year) } \\
\hline$($ Mean $\pm S D)$ & $65.57 \pm 10.15$ & $61.62 \pm 8.93$ & $0.062 *$ \\
\hline \multicolumn{4}{|l|}{ Weight (kg) } \\
\hline$($ Mean \pm SD $)$ & $84.80 \pm 7.36$ & $71.50 \pm 16.20$ & $0.052 * *$ \\
\hline \multicolumn{4}{|l|}{ Height (m) } \\
\hline$($ Mean \pm SD $)$ & $1.66 \pm 0.10$ & $1.69 \pm 0.08$ & $0.561 * *$ \\
\hline \multicolumn{4}{|l|}{ BMI $\left(\mathrm{kg} / \mathrm{m}^{2}\right)$} \\
\hline$($ Mean \pm SD $)$ & $30.88 \pm 4.42$ & $25.13 \pm 6.64$ & $0.057^{* *}$ \\
\hline \multicolumn{4}{|l|}{ Gender (\%) } \\
\hline Male & 52.4 & 66.7 & $0.266^{* * *}$ \\
\hline Female & 47.6 & 33.3 & \\
\hline \multicolumn{4}{|c|}{ Catheterization site (\%) } \\
\hline Right & 95.2 & 92.9 & $>0.05 * * *$ \\
\hline Left & 4.8 & 7.1 & \\
\hline \multicolumn{4}{|c|}{ Visualization of vein (\%) } \\
\hline Easy & 97.6 & 96.2 & $>0.05 * * *$ \\
\hline Moderate & 2.4 & 3.8 & \\
\hline Difficult & 0 & 0 & \\
\hline Impossible & 0 & 0 & \\
\hline \multicolumn{4}{|c|}{ Visualization of needle (\%) } \\
\hline Easy & 26.2 & 14.35 & \\
\hline Moderate & 54.8 & 45.2 & $0.101^{* * *}$ \\
\hline Difficult & 16.7 & 26.2 & \\
\hline Impossible & 2.4 & 14.35 & \\
\hline
\end{tabular}

SD: Standard deviation; *Independent Samples T Test; **Mann-Whitney Rank Sum Test; ${ }^{* * *}$ Chi-sqare test; Percantages are expressed as within groups.

The mean time of catheterization was $52.00 \pm 70.18$ seconds in the oblique approach group and $40.76 \pm 49.30$ seconds in the short-axis group. The median time of catheterization was 33 seconds in the oblique approach group and 30 seconds in short-axis groups. There was no statistically significant difference between the groups (Table 2).

The mean number of needle puncture attempts for successful catheter placement was $1.21 \pm 0.61$ in the oblique approach and $1.12 \pm 0.50$ in the short-axis group. All catheterization attempts were successful. In the oblique approach group, 36 patients were successfully cannulated at first attempt, four patients were cannulated at the second attempt, one patient was cannulated at the third attempt and one patient was cannulated at 
TABLE2. Comparison of outcomes between groups in terms of catheterization time, puncture attempts and complications during the catheterization procedure

\begin{tabular}{c} 
Group \\
\hline Oblique
\end{tabular}

\begin{tabular}{|c|c|c|c|}
\hline \multicolumn{4}{|l|}{ Catheterization time (seconds) } \\
\hline Mean \pm SD & $52.00 \pm 70.18$ & $40.76 \pm 49.30$ & $0.181^{* *}$ \\
\hline \multicolumn{4}{|l|}{ Number of needle insertion attempts } \\
\hline Mean \pm SD & $1.21 \pm 0.61$ & $1.12 \pm 0.50$ & $0.722 * * *$ \\
\hline $1(\%)$ & 85.7 & 92.9 & \\
\hline $2(\%)$ & 9.5 & 4.8 & \\
\hline $3(\%)$ & 2.4 & 0 & \\
\hline $4(\%)$ & 2.4 & 2.4 & \\
\hline \multicolumn{4}{|l|}{ Complications } \\
\hline Carotid puncture (\%) & 0 & 0 & $>0.05^{* * *}$ \\
\hline Pneumothorax (\%) & 0 & 0 & \\
\hline Hemothorax (\%) & 0 & 0 & \\
\hline Hematoma (\%) & 2.4 & 0 & \\
\hline Bleeding (\%) & 2.4 & 4.8 & \\
\hline Frequency of venous trombosis (\%) & 7.2 (1 right, 2 left) & 2.4 (right) & $0.752 * * *$ \\
\hline
\end{tabular}

SD: Standard deviation; ${ }^{* *}$ Mann-Whitney Rank Sum Test; ${ }^{* * *}$ Chi-sqare test; Percantages are expressed as within groups.

the fourth attempt. In the short-axis group, 39 patients were successfully cannulated at the first attempt, two patients were cannulated at the second attempt, and one patient was cannulated at the fourth attempt. The results did not differ significantly (Table 2).

Visualization of the IJV was easy in 41 patients and moderate in one patient in the oblique approach group. The IJV was visualized easily in 40 patients and moderately in two patients in the short-axis group. The results were similar between groups (Table 1 ). Visualization of the needle was classified as easy in 11 patients, moderate in 23 patients, difficult in seven patients and was impossible in one patient in the oblique approach group. Visualization of the needle was easy in six patients, moderate in 19 patients, difficult in 11 patients and was impossible in six patients in the short-axis group. These results revealed an improved visualization of the needle using the oblique approach; however, no significant difference was detected (Table 1).

The number of mechanical complications was minor. Totally, two mechanical complications occurred in each group. Bleeding from the insertion site was the most frequent complication, which developed in two patients in the short-axis group and in one patient in the oblique approach group. One small hematoma occurred in the oblique approach group. None of the patients developed a carotid puncture, pneumothorax, or hemothorax. The overall complication rates were similar between groups (Table 2).

\section{DISCUSSION}

The results of the present study suggest that the oblique approach with needle in-plane technique is a safe and effective technique. This approach may be a strong alternative to the classical short-axis technique. Oblique approach (needle in-plane) and short-axis (needle out of the plane) techniques did not differ concerning catheterization times, number of puncture attempts and frequency of complications. Although not proved statistically, the oblique approach ensured improved visibility of the needle during puncture.

There is a consensus in the literature that ultrasound guidance is mandatory for central catheterization procedures. A meta-analysis demonstrated that US guidance for IJV puncture clearly reduces the number of complications, failures and time required for insertion $[1,11]$. However, there is a controversy in which the US guided 
technique is optimal or best for reducing the time for catheterization, number of puncture attempts and mechanical complications. In previous prospective studies, short-axis, long-axis and oblique techniques have been evaluated for internal jugular vein catheterization.

In the studies that human subject was not evaluated, many of them revealed no significant difference in the number of needle punctures and time for catheterization between the long-axis and short-axis approach for internal jugular vein catheterization. Long-axis view was associated with fewer needle redirections and improved visibility of the needle $[12,13]$. Novice US users among residents completed the procedure more rapidly when using the short-axis approach without a significant difference between the LA and SA views in the number of puncture attempts and needle redirections [14].

In human studies, Chittoodan et al. demonstrated higher first-pass success and fewer needle redirections in the short-axis group (among experienced) as compared to the long-axis approach and comparable procedure time in both groups [15]. Tammam et al. found no significant difference between the short-axis and long-axis approaches [16].

After the introduction of the lateral oblique visualization of the IJV by Phelan and Hagerty [10] for ultrasound-guided IJV catheterization, a limited number of prospective studies was conducted. Wilson et al. compared the rates of posterior wall puncture and could not demonstrate a difference between the short-axis and oblique-axis approaches in their study [17]. Batllori et al. found that lateral-to medial oblique and short-axis techniques had higher first needle pass success and required fewer needle passes and less time when compared with the long-axis approach [18]. However, they did not find any difference between the short-axis and long-axis approaches concerning the number of puncture attempts, catheterization time and first needle pass success rate [18]. A different oblique approach has also been offered by DiLisio and Mittnacht in which needle insertion is performed from medial-cephalad to lateral-caudad direction [19]. However, the oblique position of the probe in the medial oblique approach may complicate needle manipulations and redirections because of the mandible. A needle path from lateral to medial can also increase the risk of carotid injury [9].

The oblique approach may be the solution to the anatomical limitation of the needle entry place on the neck imposed by the footprint of the probe. Another advantage of the oblique approach is that it provides visu- alization of both the carotid artery and the jugular vein. The short-axis approach also allows simultaneous visualization of both artery and vein, but visualization and control of the tip of the needle are difficult and impossible in many cases [18-20]. We could be able to see the tip of the needle in the vein using the oblique approach, in most of the cases which ensures excellent protection of posterior wall puncture and also makes blood aspiration during the puncture out of necessity. This can give a lead to the performance of the syringe-free catheterization technique with no attached syringe. However, this technique may be considered as a more difficult procedure due to the difficulty of visualization of the entire needle and need of experience to visualize both the needle and the vein together in the plane of the ultrasound beam. Thus, the oblique approach may be preferred after gaining experience in ultrasound-guided procedures.

Complications associated with CVC insertion range from $5 \%$ to $19 \%$, which include vascular injury (arterial puncture, pseudoaneurysm, arteriovenous fistula), hematoma, air embolism, pneumothorax and malposition [21]. The overall incidence of mechanical complications was lower in our study than previous ultrasound-guided studies. Our mechanical complications were minor, such as minor bleeding from the insertion site and a minor hematoma in one patient. Carotid artery puncture may occur in $1.1 \%$ to $1.7 \%$ and up to $4 \%$ of cases despite the use of ultrasound guidance $[13,19]$. Improved visualization of the needle tip and real-time visualization of both the needle and the carotid artery may reduce these complications and should be investigated in future studies.

Limitations of our study include that the physician performing the punctures was not blinded to the hypotheses of the study. However, differences in the ability of different operators performing the two approaches could also be a possible reason for bias. Another limitation is that we did not evaluate posterior wall puncture. This is a relatively difficult complication to diagnose, which needs an extra ultrasound.

In conclusion, the oblique approach may be considered as an alternative approach when needed, which ensures improved visualization and control of the needle. Although the short-axis approach has been traditionally the classic technique preferred by clinicians for performing and teaching catheterization of the internal jugular vein, we recommend the oblique approach as a safe and effective alternative technique. Further studies among inexperienced performers are needed to evaluate if the oblique approach is suitable as a first choice. 
Ethics Committee Approval: The Kocaeli University Clinical Research Ethics Committee granted approval for this study (date: February 24, 2015, number: KOU KAEK 2015/1).

Conflict of Interest: No conflict of interest was declared by the authors.

Financial Disclosure: The authors declared that this study has received no financial support.

Authorship Contributions: Concept - OB; Design - OB, TA, AM; Supervision - TA; Materials - OB, TA; Data collection and/or processing - OB; Analysis and/or interpretation - OB, AM; Writing - OB, TA; Critical review - TA.

\section{REFERENCES}

1. Hind D, Calvert N, McWilliams R, Davidson A, Paisley S, Beverley C, et al. Ultrasonic locating devices for central venous cannulation: metaanalysis. BMJ 2003;327:361. [CrossRef]

2. Al Sofyani K, Julia G, Abdulaziz B, Yves CJ, Sylvain R. Ultrasound guidance for central vascular access in the neonatal and pediatric intensive care unit. Saudi J Anaesth 2012;6:120-4. [CrossRef]

3. Shojania KG, Duncan BW, McDonald KM, Wachter RM, Markowitz AJ. Making health care safer: a critical analysis of patient safety practices. Evid Rep Technol Assess (Summ) 2001:i-x, 1-668.

4. National Institute for Clinical Excellence. Guidance on the Use of Ultrasound Location Devices for Placing Central Venous Catheters. Technology Appraisal Guidance No 49. September 2002 Available at: www.nice.org.uk. Accessed Dec 25, 2019.

5. Froehlich CD, Rigby MR, Rosenberg ES, Li R, Roerig PL, Easley KA, et al. Ultrasound-guided central venous catheter placement decreases complications and decreases placement attempts compared with the landmark technique in patients in a pediatric intensive care unit. Crit Care Med 2009;37:1090-6. [CrossRef]

6. Wu SY, Ling Q, Cao LH, Wang J, Xu MX, Zeng WA. Real-time twodimensional ultrasound guidance for central venous cannulation: a meta-analysis. Anesthesiology 2013;118:361-75. [CrossRef]

7. Turker G, Kaya FN, Gurbet A, Aksu H, Erdogan C, Atlas A. Internal jugular vein cannulation: an ultrasound-guided technique versus a landmark-guided technique. Clinics (Sao Paulo) 2009;64:989-92. [CrossRef]

8. Blaivas M, Adhikari S. An unseen danger: frequency of posterior vessel wall penetration by needles during attempts to place internal jugu- lar vein central catheters using ultrasound guidance. Crit Care Med 2009;37:2345-9. [CrossRef]

9. Nomura JT, Hyde RJ, Schmier CA, Sierzenski PR. Regarding the oblique view for central venous access. J Emerg Med 2011;40:331-2.

10. Phelan M, Hagerty D. The oblique view: an alternative approach for ultrasound-guided central lineplacement. J Emerg Med 2009;37:403-8.

11. Randolph AG, Cook DJ, Gonzales CA, Pribble CG. Ultrasound guidance for placement of central venous catheters: a meta-analysis of the literature. Crit Care Med 1996;24:2053-8. [CrossRef]

12. Vogel JA, Haukoos JS, Erickson CL, Liao MM, Theoret J, Sanz GE, et al. Is long-axis view superior to short-axis view in ultrasound-guided central venous catheterization? Crit Care Med 2015;43:832-9. [CrossRef]

13. Stone MB, Moon C, Sutijono D, Blaivas M. Needle tip visualization during ultrasound-guided vascular access: short-axis vs long-axis approach. Am J Emerg Med 2010;28:343-7. [CrossRef]

14. Blaivas M, Brannam L, Fernandez E. Short-axis versus long-axis approaches for teaching ultrasound-guided vascularaccess on a new inanimate model. Acad Emerg Med 2003;10:1307-11. [CrossRef]

15. Chittoodan S, Breen D, O’Donnell BD, Iohom G. Long versus short axis ultrasound guided approach for internal jugular vein cannulation: a prospective randomised controlled trial. Med Ultrason 2011;13:21-5.

16. Tammam TF, El-Shafey EM, Tammam HF. Ultrasound-guided internal jugular vein access: comparison between short axis and long axis techniques. Saudi J Kidney Dis Transpl 2013;24:707-13. [CrossRef]

17. Wilson JG, Berona KM, Stein JC, Wang R. Oblique-axis vs, short-axis view in ultrasound-guided central venous catheterization. J Emerg Med 2014;47:45-50. [CrossRef]

18. Batllori M, Urra M, Uriarte E, Romero C, Pueyo J, López-Olaondo $\mathrm{L}$, et al. Randomized comparison of three transducer orientation approaches for ultrasound guided internal jugular venous cannulation. $\mathrm{Br}$ J Anaesth 2016;116:370-6. [CrossRef]

19. Dilisio R, Mittnacht AJ. The "medial-oblique" approach to ultrasoundguided central venous cannulation--maximize the view, minimize the risk. J Cardiothorac Vasc Anesth 2012;26:982-4. [CrossRef]

20. Resnick JR, Cydulka R, Jones R. Comparison of two transducers for ultrasound-guided vascular access in long axis. J Emerg Med 2007;33:273-6. [CrossRef]

21. Rossi UG, Rigamonti P, Tichà V, Zoffoli E, Giordano A, Gallieni M, et al. Percutaneous ultrasound-guided central venous catheters: the lateral in-plane technique for internal jugular vein access. J Vasc Access 2014;15:56-60. [CrossRef] 